\title{
System-level benefit of variable data rate in optical LEO direct-to-earth links
}

Pantelis-Daniel Arapoglou, Nicolò Mazzali

Pantelis-Daniel Arapoglou, Nicolò Mazzali, "System-level benefit of variable data rate in optical LEO direct-to-earth links," Proc. SPIE 11852, International Conference on Space Optics - ICSO 2020, 118524I (11 June 2021); doi: $10.1117 / 12.2599661$

SPIE Event: International Conference on Space Optics - ICSO 2021, 2021, Online Only 


\section{International Conference on Space Optics-ICSO 2020}

Virtual Conference

30 March-2 April 2021

Edited by Bruno Cugny, Zoran Sodnik, and Nikos Karafolas
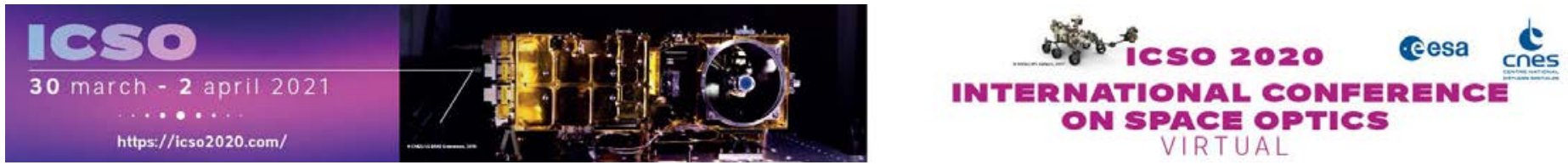

\section{System-level benefit of variable data rate in optical LEO direct-to- earth links}

\section{Cesa isoporecestings denes}




\title{
System-level Benefit of Variable Data Rate in Optical LEO Direct-to- Earth Links
}

\author{
Pantelis-Daniel Arapoglou, Nicolò Mazzali \\ European Space Agency, European Space Research \& Technology Centre (ESA/ESTEC), Radio \\ Frequency Systems Division, Keplerlaan 1, Noordwijk, The Netherlands
}

\begin{abstract}
The paper investigates the benefits of employing a Variable Data Rate (VDR) scheme in optical Low Earth Orbit (LEO) Direct-to-Earth (DTE) links at system level. In contrast to the Constant Data Rate (CDR) transmission approach adopted in current systems of the kind, the VDR scheme allows to optimize the data rate of the optical link within a satellite pass. Indeed, both the link budget and the atmospheric effects heavily depend on the satellite elevation angle, which varies significantly within a satellite pass. In order to thoroughly carry out the system level comparison between VDR and CDR, the paper first addresses all necessary aspects involved, including channel modeling, waveform and interleaver dimensioning, and link budget issues. For the scenario evaluated in the paper, the average throughput improvement of VDR versus CDR is found to be $100 \%$. The paper also addresses interesting trade-offs concerning possible concepts of operations (ConOps) of the link once the VDR is in place. This preliminary work will be followed up by the Agency with further industrialized technology developments and system refinements.
\end{abstract}

Keywords: On-Off Keying (OOK), Variable Data Rate (VDR), atmospheric turbulence, LEO direct-to-Earth.

\section{INTRODUCTION}

Currently flying and planned optical Low Earth Orbit (LEO) Direct-to-Earth (DTE) systems transmit at a constant data rate (CDR) while passing over an Optical Ground Station (OGS). However, the free space loss, the atmospheric attenuation, and the turbulence of the refraction index (causing channel fading) all exhibit a strong dependence on the satellite elevation angle. Therefore, the CDR approach leads to a significant throughput and data return loss, as it does not exploit optimally the channel capacity. Instead, a Variable Data Rate (VDR) approach, that splits the pass of the LEO satellite over the OGS in few sectors and optimizes the data rate in each of them, greatly reduces the performance gap with respect to the channel capacity.

Because of this large variability of the link budget with the elevation angle, also future radio frequency (RF)-based high data rate Earth Observation downlinks from LEO satellites (such as next generation Copernicus missions) are adopting a VDR concept based on varying the modulation order and the code rate [1]. In typical optical LEO DTE waveforms, such as the one currently defined in the Consultative Committee for Space Data Systems (CCSDS) Optical Working group referred to as Optical On-Off Keying $(\mathrm{O} 3 \mathrm{~K})$, the modulation order cannot be exploited as a degree of freedom for the VDR because it is fixed as binary. Furthermore, varying the rate of the underlying Forward Error Correction (FEC) code offers a limited dynamic range compared to the large link budget variability experienced during a satellite pass, especially for receivers based on an Avalanche Photo Diode (APD).

In this paper, the authors propose a novel way of achieving VDR in optical LEO DTE On-Off Keying (OOK) waveforms, which relies on repeating / spreading the data to achieve the most appropriate symbol rate allowed by the link budget. Among the possible different flavors of VDR [2], the authors have selected the approach that varies the symbol rate (i.e., the channel data rate) only by means of baseband operations in the digital domain and without any modification to the available transmitter and receiver hardware.

After providing an overview of the VDR concept in Section 2, the paper shall engage in a system analysis of the benefits in terms of average throughput that the VDR can offer compared to the CDR transmission. For this purpose, a link budget assessment is carried out for a particular OGS in Southern Italy, employing an APD receiver, and taking into account atmospheric channel effects, whose assumptions are explained in Section 3. 
To assess the VDR data return, detailed physical layer simulations were performed. State-of-the-art FEC coding and channel interleaving were included to cope with both thermal and shot noises (due to the APD-based receiver), as well as with the channel fading. The resulting waveform and its performance simulations are illustrated in Section 4.

The results of the physical layer simulator are fed back to the link budget. This allowed for a complete system analysis offering important insights about the interplay between the size of the channel interleaver and the fading statistics. The overall system level benefit of VDR versus CDR is assessed in Section 5, where the numerical results are presented in terms of average system throughput.

The provided system analysis helps to understand whether the advantages of the VDR at system level are attractive enough to justify the additional digital signal processing and complexity required especially on the receiver side. As this system level benefit assessment for the VDR is among the first of its kind, there is the need to further investigate specific technological aspects, as well as to identify its strengths and weaknesses. This last task is carried out in Section 6.

\section{THE VARIABLE DATA RATE CONCEPT}

The proposed VDR concept applies to a physical layer employing an OOK modulation, which due to its simplicity is typically used in free-space optical links [3], [4] and especially in low complexity optical LEO-DTE communications [5]. OOK is also the modulation format of choice in the ongoing CCSDS standardization of the optical LEO-DTE physical layer, referred to as $\mathrm{O} 3 \mathrm{~K}$ [6]. In particular, to cater for a variety of system configurations, the O3K draft standard allows for a wide range of slot widths that correspond to symbol rates from around $1.2 \mathrm{Msym} / \mathrm{s}$ to $10 \mathrm{Gsym} / \mathrm{s}$, as listed in Table 1.

Table 1. Symbol rates defined in the CCSDS O3K LEO-DTE physical layer specification.

\begin{tabular}{|c|c|c|c|c|c|}
\hline $\begin{array}{c}\text { Slot } \\
\text { widths }\end{array}$ & $0.1 \mathrm{~ns}$ & $0.2 \mathrm{~ns}$ & $0.4 \mathrm{~ns}$ & $\ldots$ & $819.2 \mathrm{~ns}$ \\
\hline $\begin{array}{c}\text { Symbol } \\
\text { Rates }\end{array}$ & $10 \mathrm{Gsym} / \mathrm{s}$ & $5 \mathrm{Gsym} / \mathrm{s}$ & $2.5 \mathrm{Gsym} / \mathrm{s}$ & $\ldots$ & $1.2 \mathrm{Msym} / \mathrm{s}$ \\
\hline
\end{tabular}

Despite this wide range of symbol rates, the Concept of Operations (ConOps) for optical LEO-DTE links is to select only one of these symbol rates and transmit at a CDR during the pass over the OGS. This approach is clearly suboptimal: if the symbol rate is chosen so to close the link under the worst channel conditions experienced during a pass, the excess of channel capacity available in many portions of the pass (where the channel conditions are better) is not exploited. Conversely, if the symbol rate is chosen according to the best channel conditions, then the link will likely not close when the channel conditions are worse, reducing the duration of the transmission window. Instead, the VDR-based ConOps relies on selecting the optimal symbol rate during the pass in a pre-programmed manner, e.g., by employing 6 different data (symbol) rates in 6 sectors of the pass.

The proposed method to realize the VDR is to repeat or spread the data symbols to the highest possible chip rate the hardware (HW) can support, where by HW we mostly refer to the laser pulse width at the transmitter and the analog bandwidth of the receiver. Hence, the proposed VDR scheme allows to change the symbol rate by operating in the digital domain on the baseband signal, keeping the HW untouched.

An illustrative example of how the proposed VDR scheme acts on the symbol (or, equivalently, coded bit) sequence is provided in Fig. 1, where it is assumed that the system's HW can support up to a 0.4 ns slot width (corresponding to a chip rate of $2.5 \mathrm{Gchip} / \mathrm{s}$ ). Assuming that the system intends to transmit at a symbol rate of $625 \mathrm{Msym} / \mathrm{s}$ (corresponding to a slot width of $1.6 \mathrm{~ns}$ ) as shown in Fig. 1a, the proposed VDR scheme will employ a repetition factor equal to four (4). As it is clear from the figure, the faster chip sequence in Fig. 1b conveys the same information as the slower binary symbol sequence is Fig. 1a. It is worth noting that, conceptually, the symbol repetition shown in Fig. 1 may be replaced by symbol spreading. 


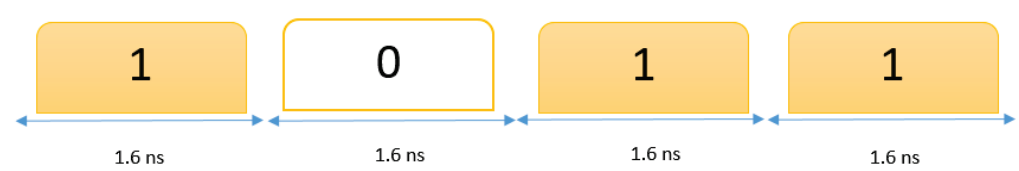

(a)

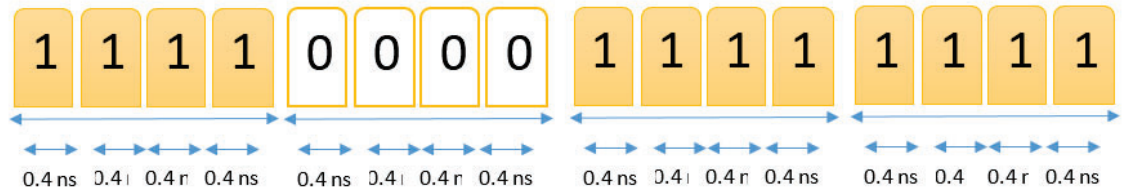

(b)

Figure 1. Example illustration of the VDR repetition/spreading concept. Initial non-spread CDR symbol sequence (a) and repeated VDR chip sequence employing a repetition factor of four (4) and offering the same information (b).
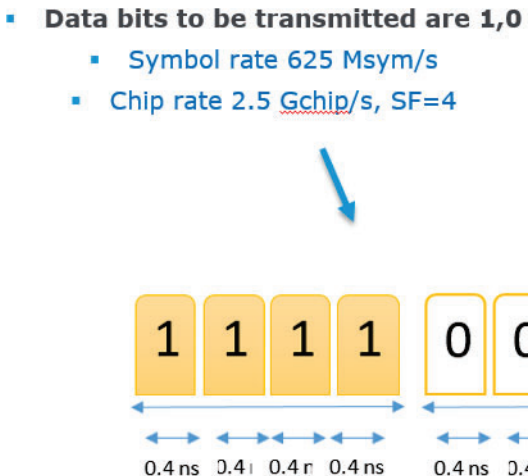

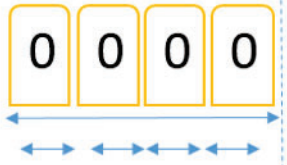

$\begin{array}{llll}0.4 \mathrm{~ns} & 0.4 & 0.4 \mathrm{r} & 0.4 \mathrm{~ns}\end{array}$

\section{- Data bit sequence is again 1,0}

- Better link budget allows to increase the Symbol rate to $1.25 \mathrm{Gsym} / \mathrm{s}$

- Chip rate is again $2.5 \mathrm{Gchip} / \mathrm{s}, \mathrm{SF}=2$
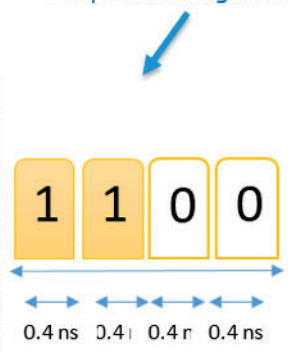

Data rate switch

Figure 2. Example of VDR symbol (data) rate switching.

Fig. 2 shows an example of how the VDR symbol rate is seamlessly adapted at a point in time when the link budget changes. In particular, the figure shows that the chip rate always remains the same $(2.5 \mathrm{Gchip} / \mathrm{s})$, but at the boundary of the data rate change the spreading/repetition factor is halved and the resulting symbol rate is doubled. Clearly, the opposite change occurs when the link budget gets worse.

Many aspects are simplified for illustrative purposes in the example in Figs. 1 and 2. There are many possible ways to implement the symbol rate adaptation, either with a particular spreading sequence or with mere repetitions. However, such detailed waveform aspects are beyond the scope of this paper, which focuses on making a first-hand assessment of the VDR system benefits in terms of average throughput compared to a CDR approach. More details on the design of the VDR transmitter in terms of spreading/repetition, framing, and receiver synchronization can be found in [7].

\section{CHANNEL MODELING AND LINK VARIABILITY}

To carry out the system level assessment, the optical LEO-DTE channel needs to be properly modeled, and in particular its elevation dependency, which is the key dimension exploited by the VDR. The underlying assumption of the paper is that the optical LEO-DTE link is cloud free, so not impacted by cloud blockage. This assumption relies on the spatial diversity guaranteed by the optical ground station network, so that cloud-free line-of-sight (CFLOS) conditions are ensured [8]. Under CFLOS, the main atmospheric effects that degrade the LEO-DTE link are:

- Atmospheric attenuation: to model the atmospheric transmittance, Beer's law [9] is employed. 
- Atmospheric turbulence due to changes in the refraction index: to model the atmospheric turbulence (equivalently called fading in the paper), the modified version of the Hufnagel-Valley model is used to represent the refractive index structure constant. Then, for the fading statistics, the weak turbulence regime is assumed, where the irradiance distribution may be approximated by a lognormal model [10]. It should be noted that, although the lognormal model is well established, it is of general applicability and, hence, it may lose in terms of accuracy at very low elevation angles (e.g. 5 deg.), where the gamma-gamma model may work better [3].

- On top of the fading, the atmospheric turbulence also degrades the spatial coherence of the laser beam, leading to a single mode fiber coupling loss, modelled according to [11].

- No pointing errors are considered.

Among the effects mentioned above, the one degrading the LEO-DTE link the most is the atmospheric turbulence. On top of its statistical model, which is needed for the link budget calculation, a dedicated tool required to synthesize a fading time series for testing the waveform performance against fading at various elevation angles.

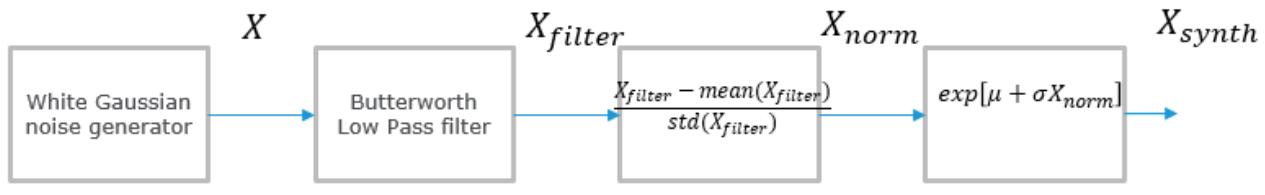

Figure 3. Atmospheric turbulence synthesizer.
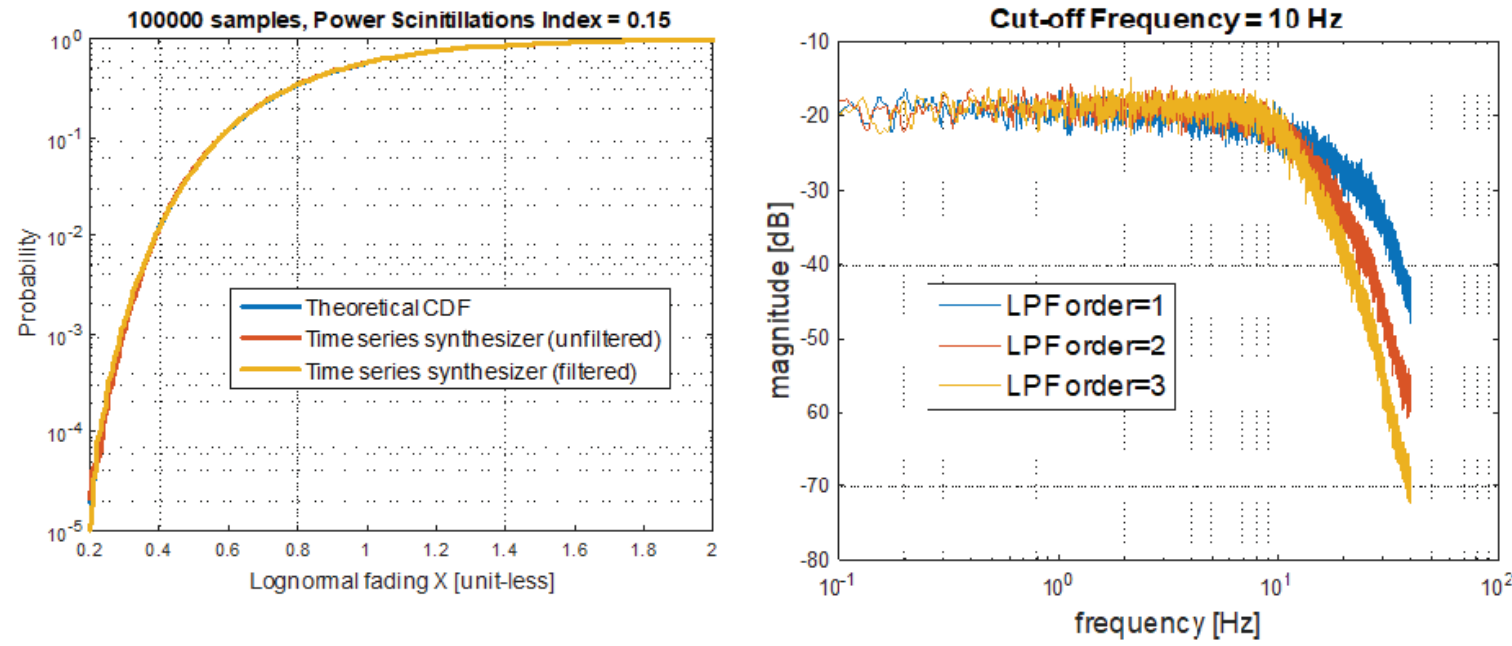

Figure 4. Validation of atmospheric turbulence synthesizer. First order statistics (left) and time series spectrum (right).

The structure of such synthesizer is described in Fig. 3 in the form of a block diagram, and is similar to the methodology presented in [12] for generating lognormal time series. First, a white Gaussian generator is used to generate independent samples that are passed through a Butterworth low pass filter (LPF) for controlling the fading dynamics. This requires as input the cut-off (Greenwood) frequency and the filter order. The LPF changes the standard deviation of the white Gaussian process, therefore the following block in the scheme is necessary to renormalize the filtered Gaussian process. Finally, the desired statistics of the time series and corresponding fading are induced by assuming a power scintillation index $\sigma_{P}^{2}$. This index is then used in the following expressions for the variance and the mean of the lognormal distribution, respectively:

$$
\begin{gathered}
\sigma^{2}=\ln \left(\sigma_{\mathrm{P}}^{2}+1\right) \\
\mu=-\sigma^{2} / 2 \text { for }\left\langle P_{R x}\right\rangle=1
\end{gathered}
$$

where $\left\langle P_{R x}\right\rangle$ is the average received optical power. It is worth noting that the final output of the synthesizer, $X_{\text {synth }}$ must have unit mean in order to make the fading time series independent of link budget. Fig.4 carries out a validation of the 
atmospheric turbulence synthesizer: in the left-hand figure the first order statistics show that analytical and simulated results match perfectly, and in the right-hand figure the power spectrum is plotted for different values of the filter order.

To understand the motivations for adopting a VDR transmission approach, it is important to get an understanding of the variability range of the channel effects depending on the elevation angle. Table 2 is representative of an OGS located in Messina, Italy, (latitude $=38.1833^{\circ} \mathrm{N}$, longitude $=15.55^{\circ} \mathrm{E}$, altitude $=507 \mathrm{~m}$ ) that is used throughout the paper. The OGS receives data from a LEO satellite on a polar orbit at $693 \mathrm{~km}$ altitude, very similar in orbital characteristics to Sentinel 1 (https://sentinel.esa.int/web/sentinel/missions/sentinel-1). Every pass of the LEO over the OGS is split into six (6) sectors of equal duration that correspond to the elevation ranges [5-7], [7-10], [10-15], [15-22], [22-37], [37-90] degrees. This convenient way of splitting the pass allows us to study the variability of the channel including all the effects in Table 2. The sectors are also used later in the paper for selecting the appropriate symbol rate according to the VDR scheme. As expected from Table 2, channel effects are harsher in the lower elevation ranges and become more benign as the elevation angle increases. It is noted that the wavelength used to derive the entries in Table 2 is $1550 \mathrm{~nm}$. A typical size for the receiver aperture is included in the table as it is required for calculating the fading due to atmospheric turbulence, the statistics of which presume a link availability of $99.9 \%$.

Table 2. Channel effects variability depending on the elevation angle.

\begin{tabular}{|c|c|c|c|c|c|c|c|}
\hline Sector\# & 1 & 2 & 3 & 4 & 5 & 6 & \\
\hline elevation range (deg) & $5-7$ & $7-10$ & $10-15$ & $15-22$ & $22-37$ & $37-90$ & 90 \\
\hline ref elevation angle (deg) & 5 & 7 & 10 & 15 & 22 & 37 & 90 \\
\hline$\%$ per sector & 16.67 & 16.67 & 16.67 & 16.67 & 16.67 & 16.67 & \\
\hline receiver aperture $(\mathrm{cm})$ & 40 & 40 & 40 & 40 & 40 & 40 & 40 \\
\hline scintillation index & 1.1881 & 0.6113 & 0.2899 & 0.1035 & 0.0369 & 0.0096 & 0.0058 \\
\hline Fried parameter $(\mathrm{cm})$ & 6.5374 & 7.8875 & 9.5499 & 12.1765 & 15.2995 & 20.3344 & 21.8664 \\
\hline $\begin{array}{l}\text { Greenwood (cut-off) } \\
\text { frequency }(\mathrm{Hz})\end{array}$ & 88 & 71.3 & 57 & 43.7 & 33 & 24 & 22 \\
\hline $\begin{array}{l}\text { atmospheric attenuation } \\
\text { (dB) }\end{array}$ & 2.61 & 1.89 & 1.36 & 0.90 & 0.62 & 0.38 & 0.32 \\
\hline scintillation loss (dB) & 15.22 & 11.67 & 8.66 & 5.73 & 3.79 & 2.21 & 1.81 \\
\hline fiber coupling loss (dB) & 13.93 & 13.22 & 12.69 & 12.22 & 11.94 & 11.72 & 11.66 \\
\hline
\end{tabular}

Table 3. Link variability over the LEO satellite pass.

\begin{tabular}{|c|c|c|c|c|c|c|c|c|}
\hline elevation (deg) & 5.00 & 7.00 & 10.00 & 15.00 & 22.00 & 37.00 & 90.00 & \\
\hline satellite altitude $(\mathrm{km})$ & 693.00 & 693.00 & 693.00 & 693.00 & 693.00 & 693.00 & 693.00 & \\
\hline Earth radius $(\mathrm{km})$ & 6376.00 & 6376.00 & 6376.00 & 6376.00 & 6376.00 & 6376.00 & 6376.00 & \\
\hline link range $(\mathrm{km})$ & 2546.91 & 2372.75 & 2139.85 & 1819.73 & 1487.37 & 1066.02 & 693.00 & \\
\hline Free space loss (dB) & 266.3 & 265.7 & 264.8 & 263.4 & 261.6 & 258.7 & 255.0 & 11.3 \\
\hline atmospheric attenuation ( $\mathrm{dB}$ ) & 2.61 & 1.89 & 1.36 & 0.90 & 0.62 & 0.38 & 0.32 & 2.3 \\
\hline scintillation index & 1.1881 & 0.6113 & 0.2899 & 0.1035 & 0.0369 & 0.0096 & 0.0058 & \\
\hline scintillation loss $(\mathrm{dB})$ & 15.22 & 11.67 & 8.66 & 5.73 & 3.79 & 2.21 & 1.81 & 13.4 \\
\hline fiber coupling loss (dB) & 13.93 & 13.28 & 12.69 & 12.22 & 11.94 & 11.72 & 11.66 & 2.26 \\
\hline
\end{tabular}

Table 3 then groups all the varying factors in the range between the minimum $\left(5^{\circ}\right)$ and maximum $\left(90^{\circ}\right)$ elevation angle of the pass, that are the free space loss, the atmospheric attenuation, the scintillation loss, and the fiber coupling loss. The last column of Table 3 shows in red the variability ranges of each quantity, computed as the difference between the corresponding maximum and minimum values. By summing up all these ranges, the total dynamic range for this link results to be $29.3 \mathrm{~dB}$. This is the dynamic range that the VDR aims at exploiting in order to bring the system throughput closer to its capacity limits.

\section{WAVEFORM ASPECTS AND PERFORMANCE}

The previous section testified to the fact that the varying elevation angle of a LEO-DTE link contributes to a significant dynamic range that the waveform needs to cope with. The main characteristics of the waveform that are considered in this paper are the following: 
- $\quad$ Binary modulation, in particular a non-return to zero (NRZ) pulsed OOK modulation [6].

- State-of-the-art FEC scheme with soft decision decoding belonging to the Turbo family. Specifically, a single code rate was selected for the serial convolutional concatenated code (SCCC) described in [13], which is an existing CCSDS standard for RF LEO-DTE systems. The choice of the particular FEC coding scheme is immaterial in the study of the VDR: any high performing coding scheme would indeed lead to similar conclusions.

- Convolutional channel interleaver. Following the SCCC encoder, a channel interleaver that is much longer than the channel coherence time is employed. This is a typical operation in slow fading channels aiming at breaking the temporal coherence of the fading process, which may affect a large number of consecutive codewords. After deinterleaving at the receiver, the bits in a codeword are affected by uncorrelated fading realizations. This allows the FEC to perform properly because the bits affected by a long deep fade event would not form a portion of a single codeword (i.e., an error burst, typically causing the decoding to fail), but they would be dispersed across multiple codewords. A detailed investigation of the channel interleaver sizing can be found in [14].

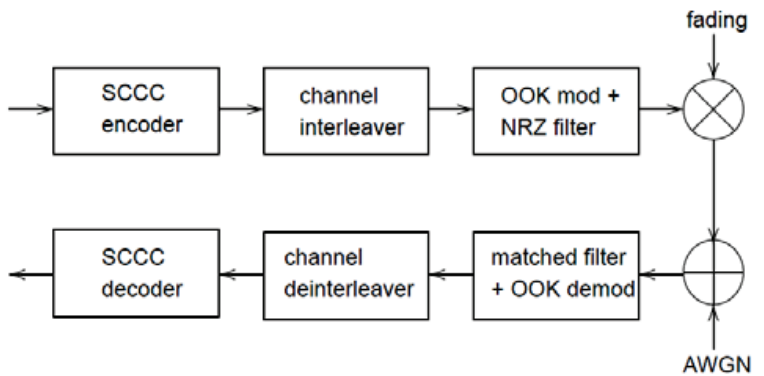

Figure 5. Block diagram of waveform simulator.

It is worthwhile mentioning that the above transmitter elements are also present in the specification of the CCSDS O3K waveform, which is under definition at the time of writing this paper. The transmitter features above, as well as their receiver counterparts, are depicted in Fig. 5, where the block diagram of the in-house waveform simulator used in this paper is presented. In low-complexity optical LEO DTE systems, it is very typical to use a receiver having an avalanche photodiode (APD) [15]. In this case, both shot noise and thermal noise are important for the detection statistics and are represented in Fig. 5 by an Additive White Gaussian Noise (AWGN) process. The fading block in Fig. 5 realizes the atmospheric turbulence samples that were generated via the process described in Section 3 and Fig. 3.

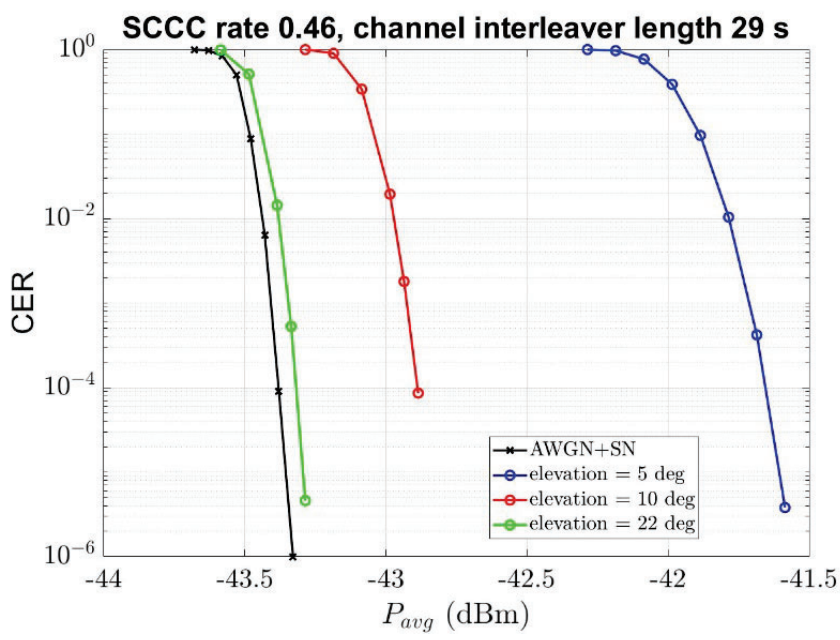

Figure 6. Waveform performance over fading at various elevation angles $\left(5^{\circ}, 10^{\circ}, 22^{\circ}\right)$. Channel interleaver length is $29 \mathrm{~s}$. 
It is not possible to evaluate the system performance of VDR without accounting for the waveform performance. Differently than under AWGN conditions, the performance over a fading channel significantly depends on the interleaver sizing. In Section 3, a 29.3 dB dynamic range due to the elevation variability was established for a particular OGS location and system scenario. However, the question is how this dynamic range is affected by the length of the channel interleaver. Toward this, we employ the waveform simulator for an SCCC code rate of 0.46 in three elevation angle scenarios $\left(5^{\circ}, 10^{\circ}\right.$ and $22^{\circ}$ ), leading respectively to three different levels of fading severity. For each fading scenario, the performance for three interleaver lengths were assessed. First, in Fig. 6 we use an unrealistically long interleaver of $29 \mathrm{~s}$ in order to find an upper bound of the Codeword Error Rate (CER) performance. The figure includes as a reference the non-faded AWGN CER (including shot noise). Rather than looking at the absolute values of the $\mathrm{x}$-axis, which corresponds to the average received optical power, the relative difference with respect to the AWGN curve should be considered. It can be seen that, even for the worst case of fading (elevation angle equal to $5^{\circ}$ ), the loss over the pure AWGN curve is merely $1.6 \mathrm{~dB}$, whereas for elevations from $22^{\circ}$ upwards, it is negligible.

Next, in Fig. 7 a channel interleaver length of $450 \mathrm{~ms}$ is selected. This is a more realistic value in terms of on-board hardware implementation. The worst case fading in this case (i.e., $5^{\circ}$ ) leads to a loss compared to AWGN of $2.6 \mathrm{~dB}$. The CER curve for $22^{\circ}$ elevation is about $0.25 \mathrm{~dB}$ worse than AWGN.

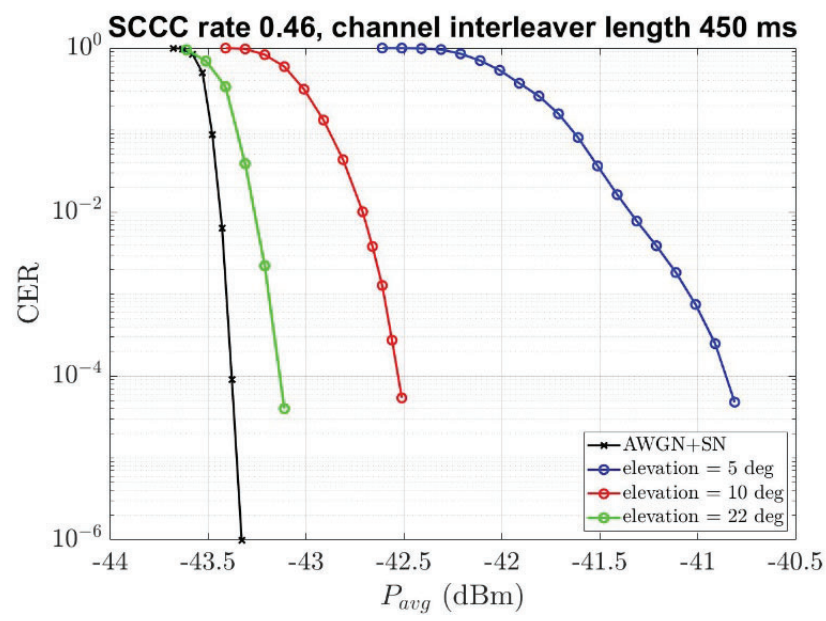

Figure 7. Waveform performance over fading at various elevation angles $\left(5^{\circ}, 10^{\circ}, 22^{\circ}\right)$. Channel interleaver length is $450 \mathrm{~ms}$.

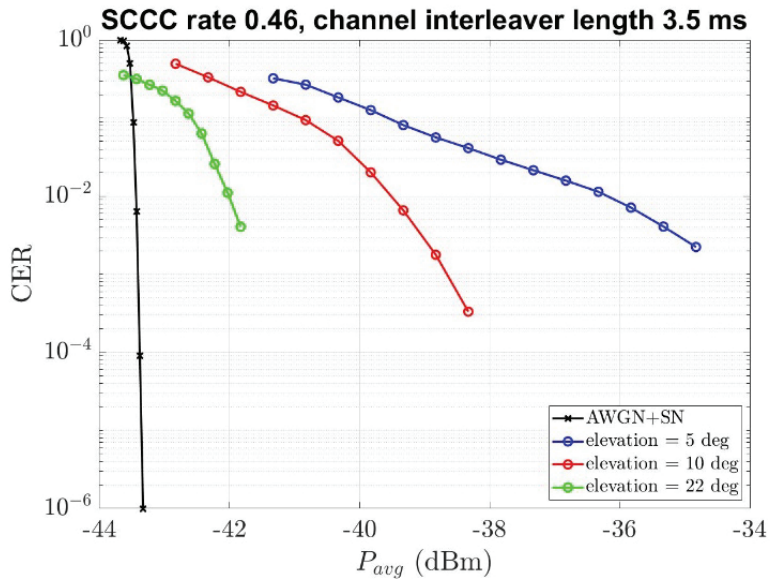

Figure 8. Waveform performance over fading at various elevation angles $\left(5^{\circ}, 10^{\circ}, 22^{\circ}\right)$. Channel interleaver length is $3.5 \mathrm{~ms}$. 
Finally, the opposite extreme case of a very short interleaver length of $3.5 \mathrm{~ms}$ was considered. Fig. 8 shows that under these conditions the decoder does not work at all, as the selected interleaver is much shorter than the fading coherence time.

Out of the waveform performance simulations shown in this section, the fundamental conclusion is that, even for a very low elevation angle of $5^{\circ}$, a long channel interleaver manages to recover all the fading losses with a reasonable power penalty compared to AWGN. Ignoring the unrealistically long interleaver of $29 \mathrm{~s}$ and the too short one of $3.5 \mathrm{~ms}$, the power penalty caused by the fading is well within $3 \mathrm{~dB}$ for a reasonably sized interleaver of $450 \mathrm{~ms}$. In other words, by using this waveform, the total dynamic range of the link budget during a pass from the LEO satellite over the OGS is dominated by the factors others than the scintillation, and is reduced from $29.3 \mathrm{~dB}$ to $16 \mathrm{~dB}$. This conclusion seems to be in contrast with the bulk of literature for optical LEO DTE links that seem to exclude elevation angles as low as $5^{\circ}$.

Table 4. Waveform sensitivity thresholds assuming SCCC rate 0.46 with $450 \mathrm{~ms}$ interleaving.

slot widths (ns)
0.1
0.2
0.4
0.8
1.6
3.2
6.4
12.8
25.6
51.2
102.4
204.8
409.6
819.2

819.2

$\begin{gathered}\text { symbol rates } \\ \text { (Msym/s) }\end{gathered}$
10000
5000
2500
1250
625
312.50
156.25
78.13
39.06
19.53
9.77
4.88
2.44
1.22

1.22
SCCC 0.46 AWGN

\section{(dBm)}

$-43.5$

$-46.2$

$-48.9$

$-51.6$

$-54.3$

$-57$

$-59.7$

$-62.4$

$-65.1$

$-67.8$

$-70.5$

$-73.2$

$-75.9$

$-78.6$

SCCC 0.46 fading
$(\mathbf{d B m})$
-40.5
-43.2
-45.9
-48.6
-51.3
-54
-56.7
-59.4
-62.1
-64.8
-67.5
-70.2
-72.9
-75.6

In the discussion so far we have not distinguished between VDR and CDR. We have simply assumed that the VDR can transparently change its symbol rate. However, for a fixed memory of the channel interleaver, its duration will change as the symbol rate changes. Therefore, a reasonable VDR ConOps would be to fix and oversize the channel interleaver memory (in bits) based on the minimum elevation angle (i.e., $5^{\circ}$ in our analysis), without constraining its duration in seconds. For each possible slot width (or, equivalently, symbol rate) of the considered waveform (i.e., SCCC rate 0.46 with a $450 \mathrm{~ms}$ channel interleaver), conservative sensitivity thresholds for link budget purposes were adopted and reported in Table 4. Consistently with the worst case shown in Fig.7, the new sensitivity thresholds for the fading channel were obtained simply by adding $3 \mathrm{~dB}$ of additional required average power to the thresholds for the AWGN channel. These new sensitivity thresholds in Table 4 are used for the purpose of system assessment in the next section.

\section{VDR SYSTEM LEVEL ASSESSMENT}

Armed with the channel modeling and waveform performance results presented in the previous sections, we are now in a position to carry out the VDR vs. CDR system level assessment. As introduced in Section 3, the system analysis is carried out for a LEO satellite in a polar orbit and a hypothetical OGS located in Messina, Italy. The pass over the OGS is split into six sectors with equal durations as shown in Table 2, where in each of them the VDR selects an appropriate symbol rate. Instead, the CDR employs a single symbol rate for the whole pass over the OGS, whose selection is elaborated in this section. 


\subsection{Link budgets}

To evaluate the average VDR and CDR system throughput during the pass, a link budget for each of the six sectors was calculated. Apart from parameters that render the link variable with elevation (see Table 3), there are also fixed link parameters which are listed in Table 5.

Table 5. Fixed link parameters.

\begin{tabular}{|c|c|}
\hline Parameter & Value \\
\hline Wavelength & $1550 \mathrm{~nm}$ \\
\hline transmit power & $0.5 \mathrm{~W}$ \\
\hline transmit aperture & $5 \mathrm{~cm}$ \\
\hline receive aperture & $40 \mathrm{~cm}$ \\
\hline Waveform & OOK SCCC rate 0.46 \\
\hline Interleaver length & $450 \mathrm{~ms}$ \\
\hline Waveform sensitivity & AWGN threshold $+3 \mathrm{~dB}$ (see Table \\
thresholds & 4 ) \\
\hline Modem implementation loss & $2 \mathrm{~dB}$ \\
\hline Link margin & $>3 \mathrm{~dB}$ \\
\hline
\end{tabular}

For the transmit and receive aperture sizes, as well as for the transmit power, typical values were used that are representative of real satellite and ground optical terminals. The rest of the assumptions in Table 5 rely on an SCCC rate 0.46 waveform with $450 \mathrm{~ms}$ channel interleaving length. In addition to the $3 \mathrm{~dB}$ margin that is assumed over the theoretical AWGN values for the required power of each symbol rate (see Table 4), there is also a $2 \mathrm{~dB}$ margin to account for the losses when the waveform is implemented in a real modem HW compared to theory. Finally, as the symbol rate selected for each sector is pre-programmed, at least $3 \mathrm{~dB}$ of link margin are required by the link budget.

Table 6. Link budget without FEC \& interleaving.

\begin{tabular}{|c|c|c|c|c|c|c|c|}
\hline & 1 & 2 & 3 & 4 & 5 & 6 & \\
\hline elevation range & $5-7$ & $7-10$ & $10-15$ & $15-22$ & $22-37$ & $37-90$ & 90 \\
\hline wavelength $(\mathrm{nm})$ & 1550.00 & 1550 & 1550 & 1550 & 1550 & 1550 & 1550 \\
\hline elevation (deg) & 5.00 & 7.00 & 10.00 & 15.00 & 22.00 & 37.00 & 90.00 \\
\hline satellite altitude $(\mathrm{km})$ & 693.00 & 693.00 & 693.00 & 693.00 & 693.00 & 693.00 & 693.00 \\
\hline link range (km) & 2546.91 & 2372.75 & 2139.85 & 1819.73 & 1487.37 & 1066.02 & 693.00 \\
\hline Tx power (W) & 0.5 & 0.5 & 0.5 & 0.5 & 0.5 & 0.5 & 0.5 \\
\hline Tx power (dBm) & 27.0 & 27.0 & 27.0 & 27.0 & 27.0 & 27.0 & 27.0 \\
\hline Tx optical loss (dB) & 3 & 3 & 3 & 3 & 3 & 3 & 3 \\
\hline Tx aperture $(\mathrm{cm})$ & 5 & 5 & 5 & 5 & 5 & 5 & 5 \\
\hline Tx antenna gain (dB) & 99.2 & 99.2 & 99.2 & 99.2 & 99.2 & 99.2 & 99.2 \\
\hline Free space loss (dB) & 266.3 & 265.7 & 264.8 & 263.4 & 261.6 & 258.7 & 255.0 \\
\hline atmospheric attenuation (dB) & 2.6199 & 1.8961 & 1.3699 & 0.9099 & 0.6208 & 0.386 & 0.3248 \\
\hline scintillation index & 1.1801 & 0.6113 & 0.2800 & 0.1035 & 0.369 & 0.0096 & 0.0058 \\
\hline scintillation loss (dB) & 15.2252 & 11.6717 & 8.6606 & 5.7367 & 3.7952 & 2.2133 & 1.8111 \\
\hline fiber coupling loss (dB) & 13.931 & 13.2248 & 12.6942 & 12.2287 & 11.9433 & 11.7226 & 11.6679 \\
\hline cloud margin (dB) & 3 & 3 & 3 & 3 & 3 & 3 & 3 \\
\hline $\mathrm{Rx}$ aperture $(\mathrm{cm})$ & 40 & 40 & 40 & 40 & 40 & 40 & 40 \\
\hline$R x$ antenna gain (dB) & 117.1 & 117.1 & 117.1 & 117.1 & 117.1 & 117.1 & 117.1 \\
\hline Rx optical loss (dB) & 3 & 3 & 3 & 3 & 3 & 3 & 3 \\
\hline Rx optical power (dBm) & -63.8 & -58.2 & -53.2 & -48.0 & -43.7 & -38.8 & -34.5 \\
\hline
\end{tabular}

Table 7. Link budget including FEC coding and interleaving. 


\begin{tabular}{|c|c|c|c|c|c|c|}
\hline & 1 & 2 & 3 & 4 & 5 & 6 \\
\hline elevation range & $5-7$ & $7-10$ & $10-15$ & $15-22$ & $22-37$ & $37-90$ \\
\hline wavelength (nm) & 1550.00 & 1550 & 1550 & 1550 & 1550 & 1550 \\
\hline satellite altitude $(\mathrm{km})$ & 693.00 & 693.00 & 693.00 & 693.00 & 693.00 & 693.00 \\
\hline link range $(\mathbf{k m})$ & 2546.91 & 2372.75 & 2139.85 & 1819.73 & 1487.37 & 1066.02 \\
\hline Tx power (W) & 0.5 & 0.5 & 0.5 & 0.5 & 0.5 & 0.5 \\
\hline Tx optical loss (dB) & 3 & 3 & 3 & 3 & 3 & 3 \\
\hline Tx aperture $(\mathrm{cm})$ & 5 & 5 & 5 & 5 & 5 & 5 \\
\hline Tx antenna gain (dB) & 99.2 & 99.2 & 99.2 & 99.2 & 99.2 & 99.2 \\
\hline Free space loss (dB) & 266.3 & 265.7 & 264.8 & 263.4 & 261.6 & 258.7 \\
\hline atmospheric attenuation (dB) & 2.6199 & 1.8961 & 1.3699 & 0.9099 & 0.6208 & 0.386 \\
\hline scintillation index & 11881 & 0.6112 & 0.2899 & 0.1035 & 0.0369 & 0.0096 \\
\hline scintillation loss (dB) & 0 & 0 & 0 & 0 & 0 & 0 \\
\hline fiber coupling loss ( $\mathrm{dB})$ & 13.931 & 13.2240 & 12.0942 & 12.2281 & 11.9433 & 11.7226 \\
\hline cloud margin (dB) & 3 & 3 & 3 & 3 & 3 & 3 \\
\hline$R x$ aperture $(\mathrm{cm})$ & 40 & 40 & 40 & 40 & 40 & 40 \\
\hline Rx antenna gain (dB) & 117.1 & 117.1 & 117.1 & 117.1 & 117.1 & 117.1 \\
\hline Rx optical loss (dB) & 3 & 3 & 3 & 3 & 3 & 3 \\
\hline Rx optical power (dBm) & -48.6 & -46.5 & -44.6 & -42.2 & -39.9 & -36.6 \\
\hline Code rate & 0.46 & 0.46 & 0.46 & 0.46 & 0.46 & 0.46 \\
\hline Symbol rate (Msvm/s) & 312.50 & 312.50 & 625 & 1250 & 2500 & 5000 \\
\hline Required optical power (dBm) & -54 & -54 & -51.3 & -48.6 & -45.9 & -43.2 \\
\hline modem implementation loss (đob) & 2 & 2 & 2 & 2 & 2 & 2 \\
\hline Margin (dB) & 3.4 & 5.5 & 4.7 & 4.4 & 4.0 & 4.6 \\
\hline offered data rate (Mbps) & 143.8 & 143.8 & 287.5 & 575.0 & 1150.0 & 2300.0 \\
\hline
\end{tabular}

The link budgets per sector resulting from these assumptions are presented in Table 6. The effects of FEC coding and interleaving are not included in the table, which remains at the level of average received optical power. Then, in Table 7 , where the effects of FEC coding and interleaving are accounted for, the link budget also yields the selection of the symbol rate and the offered data rate. As concluded in Section 4, the long channel interleaver eradicates the effect of scintillation loss (apart from the $3 \mathrm{~dB}$ margin in the symbol rate selection) and is therefore set to $0 \mathrm{~dB}$ in Table 7.

\subsection{Average system throughput results}

After having established the link budgets for each sector, it is now possible to deduce the average system throughput for both CDR and VDR. To find the best possible CDR system throughput, Table 8 shows six results for CDR, assuming that the transmission starts in each of the six sectors. Remembering that CDR can only employ a single symbol rate throughout the pass, the aforementioned approach essentially trades a lower visibility time with a higher average throughput. Indeed, looking at the first six rows of Table 8, the best CDR throughput corresponds to initiating transmission at sector 5 and transmitting at $1150 \mathrm{Mbps}$ (see column 5 of Table 7) for $2 / 6$ of the time (i.e., during sectors 5 and 6 ), or starting at sector 6 and transmitting at $2300 \mathrm{Mbps}$ (see column 6 of Table 7) for 1/6 of the time. Both options result in an average throughput over the full pass equal to $383.3 \mathrm{Mbps}$. Interestingly, the transmitter being active for only one sector may lead to significant energy savings and battery recharge cycles. On the other hand, the VDR average throughput is computed by averaging the offered data rates across the sectors (last row of Table 7). This yields $766.7 \mathrm{Mbps}$, which is an improvement of $100 \%$ of data return compared to the best CDR option.

Table 8. Average system throughput comparison between VDR and CDR.

\begin{tabular}{|c|c|}
\hline Scheme & $\begin{array}{c}\text { Average system } \\
\text { throughput over the full } \\
\text { pass (Mbp) }\end{array}$ \\
\hline CDR, starting at Sector\#1 & 143.8 \\
\hline CDR, starting at Sector\#2 & 119.8 \\
\hline CDR, starting at Sector\#3 & 191.7 \\
\hline CDR, starting at Sector\#4 & 287.5 \\
\hline CDR, starting at Sector\#5 & 383.3 \\
\hline CDR, starting at Sector\#6 & 383.3 \\
\hline VDR & 766.7 \\
\hline
\end{tabular}




\section{DISCUSSION AND CONCLUSIONS}

The paper attempted to derive the system-level benefit of VDR in optical LEO-DTE links and to highlight whether the advantages in terms of data volume return justify the additional digital signal processing and complexity required by VDR. After presenting the VDR main concept in Section 2, the paper developed the necessary channel modelling in Section 3 and waveform performance in Section 4 to support the link budget and system analysis in Section 5. To the authors' knowledge, this comprehensive approach to the system analysis of VDR vs. CDR is the first of its kind.

The strengths of the proposed VDR concept include:

- The seamless implementation of VDR in an optical LEO-DTE link with a significant positive impact on data return.

- The offer of various data rates during a pass but also between different OGSs and/or in different passes over the same OGS.

- The implementation of VDR by using a single laser pulse width, and by operating at a fixed chip rate at the receiver.

- The adoption of the CCSDS O3K physical layer book [6] without any modifications, especially concerning the already defined symbol rates.

- The use of an optimized range of code rates from a receive photon sensitivity point of view. Indeed, despite the fact that the analysis presented in this paper was carried out with a fixed FEC code rate, nothing prevents the proposed VDR scheme to attain a finer granularity by allowing joint symbol and code rate adaptation.

The proposed VDR scheme comes also with some weaknesses that need to be further evaluated in follow-up industrial activities:

- The additional receiver complexity linked to the sampling rate (since the receiver operates at a fixed chip rate, independently of the symbol rate), and the synchronization.

- The extension of the transmission window to lower elevation angles may render the timing drift caused by the Doppler effect quite critical.

- As the noise density of a typical transimpedance amplifier (TIA) is non-uniform for varying symbol rates, there may be an additional loss when operating at a much higher chip rate compared to a single low symbol rate for CDR.

One significant finding based on the waveform simulation results is that, although the optical LEO-DTE link budget presents a very large dynamic range with respect to elevation angle (around $30 \mathrm{~dB}$ ), the use of a moderate channel interleaving along with advanced FEC coding leads to the shrinkage of this dynamic range (to about $15 \mathrm{~dB}$ ). This fact reduces the potential benefit of the VDR. Nevertheless, in our assessment the VDR allowed an improvement of the average throughput during the satellite pass of $100 \%$ with respect to the best throughput obtained with CDR under the same operational conditions. This result is of course specific to the OGS location and the symbol rate granularity that were considered. Using an adaptive sensitivity approach for the symbol rates, meaning assigning a lower fading margin for higher elevation angles, results in an improvement of the average throughput of $87.5 \%$ with respect to the throughput obtained with the CDR.

Notwithstanding some of the VDR weaknesses already identified (i.e., possible synchronization issues at low elevation, TIA noise increase, etc.), the VDR technique offers great flexibility for a better optimization of different types of optical LEO-DTE systems over different OGSs. Hence, this preliminary work will be followed up by the Agency with further industrialized technology developments and system refinements [16]. Also, the VDR is one of the candidate techniques that may be included in the CCSDS O3K coding and synchronization layer currently under definition. 


\section{REFERENCES}

[1] N. Toptsidis, P.-D. Arapoglou, M. Bertinelli, "Link adaptation for Ka band LEO Earth observation systems: A realistic performance assessment," International Journal of Satellite Communications \& Networking, vol. 30, no. 3, pp. 131-146, May/June 2012.

[2] A. Shrestha, D. Giggenbach, "Variable data rate for Optical Low-Earth-Orbit (LEO) Downlinks," Proceedings of Photonic Networks; 17. ITG-Symposium; Leipzig, Germany, 12-13 May 2016.

[3] F. Xu, A. Khalighi, P. Causse, S. Bourennane, "Channel coding and time-diversity for optical wireless links," Optics Express, vol. 17, no. 2, January 2009.

[4] K. Kiasaleh, "Receiver Architecture for Channel-Aided, OOK, APD-based FSO Communications Through Turbulent Atmosphere," IEEE Trans. Commun., vo. 63, no. 1, Jan. 2015.

[5] D. T. Nguyen, Y. Park, "Performance analysis of interleaved LDPC for optical satellite communications," Optics Communications, vol. 442, pp. 13-18, 2019.

[6] CCSDS 141.0-B-1, "Optical Communications Physical Layer," Recommended Standard + Pink Sheets for O3K, February 2020.

[7] D. Arapoglou, N. Mazzali, G. Colavolpe, L. Gaudio, "Variable Data Rate (VDR) in O3K: Initial Performance Results" ESA presentation to CCSDS Optical Working Group monthly meeting, 14 April 2020. available at https://www.researchgate.net/publication/341130282_Variable_Data_Rate_VDR_in_O3K_Initial_Performance Results

[8] N.K. Lyras, C.N. Efrem, C. I. Kourogiorgas, A. D. Panagopoulos, P.-D. Arapoglou, "Optimizing the Ground Network of MEO Optical Satellite Communication Systems" IEEE Systems Journal, vol. 14, no. 3, pp. 39683976, Sep. 2020.

[9] https://en.wikipedia.org/wiki/Beer\%E2\%80\%93Lambert law

[10]D. Giggenbach, H. Henniger, "Fading-loss assessment in atmospheric free space optical communication links with on-off keying," Optical Engineering, vol. 47, no. 4, 046001, April 2008.

[11] H. Takenaka, M. Toyoshima, and Y. Takayama, "Experimental verification of fiber-coupling efficiency for satellite-to-ground atmospheric laser downlinks," Optics Express, vol. 20, no. 14, July 2012.

[12]D. Giggenbach, A. Shrestha, F. Moll, C. Fuchs, K. Saucke, "Reference Power Vectors for the Optical LEO Downlink Channel," in IEEE International Conference on Space Optical Systems and Applications (ICSOS), 14-16 Oct. 2019.

[13]CCSDS 131.2-B-1 Recommended standard, "Flexible Advanced Coding \& Modulation Scheme for High Rate Telemetry Applications". March 2012.

[14] N. Mazzali, P.-D. Arapoglou, "Channel Interleaver Dimensioning for Optical LEO Direct-to-Earth Systems," in 10th Advanced Satellite Multimedia Systems Conference (ASMS) and 16th Signal Processing for Space Communications Workshop (SPSC), Graz, Austria, 2020.

[15] S. Poulenard, B. Gadat, J. F. Chouteau, T. Anfray, C. Poulliat, C. Jego, O. Hartmann, G. Artaud, and H. Meric "Forward error correcting code for high data rate LEO satellite optical downlinks", Proc. SPIE 11180, International Conference on Space Optics - ICSO 2018.

[16]ESA ITT 20.1TI.07 ARTES 4.0 SPL Optical Communication - ScyLight: Variable Data Rate transmitter for LEO Direct-to-Earth optical links (5G.015/SL.034) (issued). 